\title{
Process Chain Network (PCN) and Business Process Modeling Notation (BPMN): A Comparison of Concepts
}

\author{
Yahya Kazemzadeh ${ }^{1}$, Simon K. Milton ${ }^{1} \&$ Lester W. Johnson $^{2}$ \\ ${ }^{1}$ Department of Computing and Information Systems, The University of Melbourne, Melbourne, Australia \\ 2 Swinburne Business School, Melbourne, Australia \\ Correspondence: Yahya Kazemzadeh, Department of Computing and Information Systems, The University of \\ Melbourne, Parkville, VIC 3010, Australia. E-mail: yahyak@student.unimelb.edu.au \\ Received: January 21, 2015 \\ Accepted: February 5, $2015 \quad$ Online Published: February 13, 2015 \\ doi:10.5430/jms.v6n1p88 \\ URL: http://dx.doi.org/10.5430/jms.v6n1p88
}

\begin{abstract}
Process chain network (PCN) and business process modeling notation (BPMN) are two alternative ways to visualize a service delivery process. They take different perspectives towards service operations and are used in slightly different circumstances. We examine the similarities and differences between concepts in PCN and BPMN, using the method of conceptual evaluation and suggest ways that they can be used by service designers. Specifically, we conclude that whilst PCN focuses on customer-provider interaction, BPMN can depict all communications between different organizational departments, roles and systems.
\end{abstract}

Keywords: process chain network (PCN), business process modeling notation (BPMN), conceptual evaluation

\section{Introduction}

Sampson (2011, 2012b, 2012a), introduced a new service visualisation technique, namely Process-Chain-Network, abbreviated PCN. This technique provides a stable and balanced assessment of the communication between customer and service provider. PCN explicitly considers service as a type of resource/process configuration. Sampson (2012a, p.17) argues that "PCN diagrams build on the strengths of other flowcharting techniques, while emphasizing the unique conditions and design opportunities for interactive service processes". Service designers and analysts are therefore assisted by the PCN framework in analysing service operations in a systematic way.

Another process representation technique is Business Process Modelling Notation (BPMN) which is used by business process analysts to design and document intra-organizational and service processes. BPMN was first introduced in 2004 by an industry consortium (BPMI Notation Working Group), who are all tool vendors rather than end users (BPMI.org, 2004). Since that time, BPMN has become a popular standard for visualizing corporate procedures by the business community, systems analysts, and also process analysts (Recker, 2010). When one wishes to design business processes at early stages of systems development (Dijkman, Dumas, \& Ouyang, 2008), business analysis (Recker, Rosemann, Green, \& Indulska, 2011), web services (Decker, Kopp, Leymann, \& Weske, 2009) and develop service-oriented architectures (Rabhi, Yu, Dabous, \& Wu, 2006), BPMN is used.

This study examines the similarities and differences between the concepts of PCN and BPMN. Upon observing this, we can understand in which stages of service development these models can be used. Additionally, the results that we observe can assist the process of mapping one model to another and being able to use them in conjunction. Therefore, the main research question is:

What are the similarities and differences of the concepts in PCN with the concepts in BPMN?

To find the answer to the above question, we must first answer two subordinate research questions:

How well do BPMN concepts support PCN concepts?

How well do PCN concepts support BPMN concepts?

We use the method of conceptual evaluation (Kazemzadeh, Milton, \& Johnson, in press-c; Milton \& Johnson, 2012; Milton \& Kazmierczak, 2004) to answer each of the subordinate questions, thus providing an answer to the main question. This method has been further developed by Kazemzadeh, Milton, and Johnson (in press-a, in press-b) to reduce its subjectivity. We use the method of conceptual evaluation due to the fact that it was the only method we 
found in the service science literature for conducting a comparison of this kind.

We next provide a detailed explanation of the research method. Following this, both modelling formalisms are defined and briefly described. Afterwards, a conceptual assessment of BPMN against PCN and vice-versa is conducted. The findings from these evaluations and conclusions are presented next. Finally, practical implications and possible future research directions are discussed.

\section{Research Design}

Let us set A and B as the process modeling formalisms being studied. We now list the steps required to conduct the comparison:

Step 1. Define the concepts of A. These are $<\mathrm{a} 1>,<\mathrm{a} 2>, \ldots,<\mathrm{an}>$.

Step 2. Define the concepts of B. These are $<\mathrm{b} 1>,<\mathrm{b} 2>, \ldots,<\mathrm{bm}>$.

Step 3. Evaluate the concepts of B against A. Specficially concepts in turn from A will be compared to various concepts from B. We employ semiotic theories, and the idea of semantic fields covered by concepts, in order to do this. By this we mean that concepts in each modeling framework span parts of a semantic field (Eco, 1976), or conceptual plane (Cruse, 2004; Culler, 1976). Therefore, when comparing concepts from two different process modeling formalisms, we are comparing the similarities and differences of the 'semantic field' associated with each concept.

More formally, comparing concepts $<\mathrm{ai}>(\mathrm{i}=1 \ldots \mathrm{n})$, that constitue the process modeling framework A, with concepts from B, gives us three possible outcomes: full coverage, partial coverage, or no coverage of the semantic field. When the semantic field covered by concept <ai $>$ fully overlaps with the semantic field of one or a combination of more than one concepts from B, we call this full coverage. Similarly, partial overlap between the semantic field covered by concept $<\mathrm{ai}>$ can be from one or a combination of more than one concepts from B. No overlap, is where there is no coverage by $\mathrm{B}$ at all for the concept $<\mathrm{ai}>$.

Comparison proceeds by examining each concept from A and seeking concept(s) from B that provides full coverage. Failing this, partial coverage by concept(s) from B is sought. If none are found then no overlap is concluded.

Importantly, a simple concept or a minimal combination of concepts from B covering the maximum semantic field is chosen. This simple or minimal combination of concepts is called a 'supportive concept'. For example, together, as a supportive concept, the concept(s) $<\mathrm{bi}^{\prime}>, \ldots,<\mathrm{bj}^{\prime}>\left(0<\mathrm{i}^{\prime} \leq \mathrm{j}^{\prime} \leq \mathrm{m}\right)$ from $\mathrm{B}$, may provide partial or full coverage of the semantic field for the concept $<\mathrm{ai}\rangle$. For ease of expression, the supportive concept is shown as $\left\langle\mathrm{bi}^{\prime}+\ldots+\mathrm{bj}^{\prime}\right\rangle$. However, in discussion we use the word 'plus' rather than ' + '.

Generalising, it is possible to have instances in which the same semantic field, <ai $>$, is partly or fully covered by different distinct supportive concepts from B. The group of supportive concepts independently providing the same semantic coverage could be called $\mathrm{Si}(\mathrm{B})=\left\{<\mathrm{bi}^{\prime}+\ldots+\mathrm{bj}^{\prime}>, \ldots,<\mathrm{bi}^{\prime \prime}+\ldots+\mathrm{bj}^{\prime \prime}>\right\}\left(0<\mathrm{i}^{\prime} \leq \mathrm{j}^{\prime} \leq \mathrm{i}^{\prime \prime} \leq \mathrm{j}^{\prime \prime} \leq \mathrm{m}\right)$. This specifically, means $<$ ai $>$ is covered by $\left\langle\mathrm{bi}^{\prime}+\ldots+\mathrm{bj}^{\prime}>\right.$, but also independently $<\mathrm{ai}>$ is covered by $<\mathrm{bi}^{\prime \prime}+\ldots+$ bj">. This general case has not been found in practice.

If there is no coverage from $\mathrm{B}$ of the semantic field for the concept $<\mathrm{ai}>$, then $\operatorname{Si}(\mathrm{B})=\{\varnothing\}$.

Figure 1 shows the three types of results for the coverage of $<$ ai $>$ by Si(B). In figure 1 , the three symbols $(\checkmark),(\checkmark p)$, and ( $\mathbf{x})$, are used for full, partial, and no coverage respectively. These symbols are used in results tables later in the paper.

Step 4. This step is very similar to step 3, but evaluates A against concepts from B.

Step 5. This step consolidates the results and describes the implications of the overall results. Two major findings in this step may be:

a) Suppose $<$ bi $^{\prime}+\ldots+$ bj' $>$ fully covers $<$ ai $>, \ldots,<a j>$ and $<a i+\ldots+$ aj $>$ fully covers $<$ bi' $^{\prime}>, \ldots,<$ bj' $^{\prime}>$.

Then: $<b^{\prime}+\ldots+b^{\prime}>$ covers the same semantic field as $<a i+\ldots+a j>$.

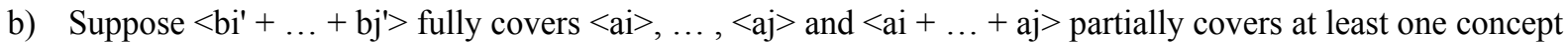
from $<\mathrm{bi}^{\prime}>, \ldots,<\mathrm{bj}^{\prime}>$.

Then: $<$ bi' $^{\prime}+\ldots+$ bj'$^{\prime}>$ has a broader semantic field than $<a i+\ldots+a j>$. 


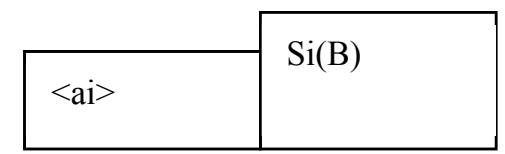

\begin{tabular}{|l|l|}
\hline$<\mathrm{ai}>$ & $\mathrm{Si}(\mathrm{B})$ \\
\hline
\end{tabular}

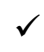

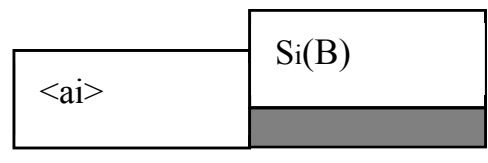

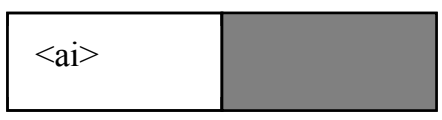

Figure 1. Degree of overlap in coverage of semantic field for $<$ ai $>$ by $\operatorname{Si}(B)$

\section{Process Chain Network (PCN)}

Figure 2 below, offers an example of a PCN schematic of a hotel stay. Such a diagram includes process entities; those that contribute to the service delivery that fulfils their needs and requirements. In the example described in this section, the hotel and the hotel guests are those that would be identified as process entities. The arrows within the figure show the relationship and connection between the different activities. These are named process step and their sequence of implementation is presented by the arrows.

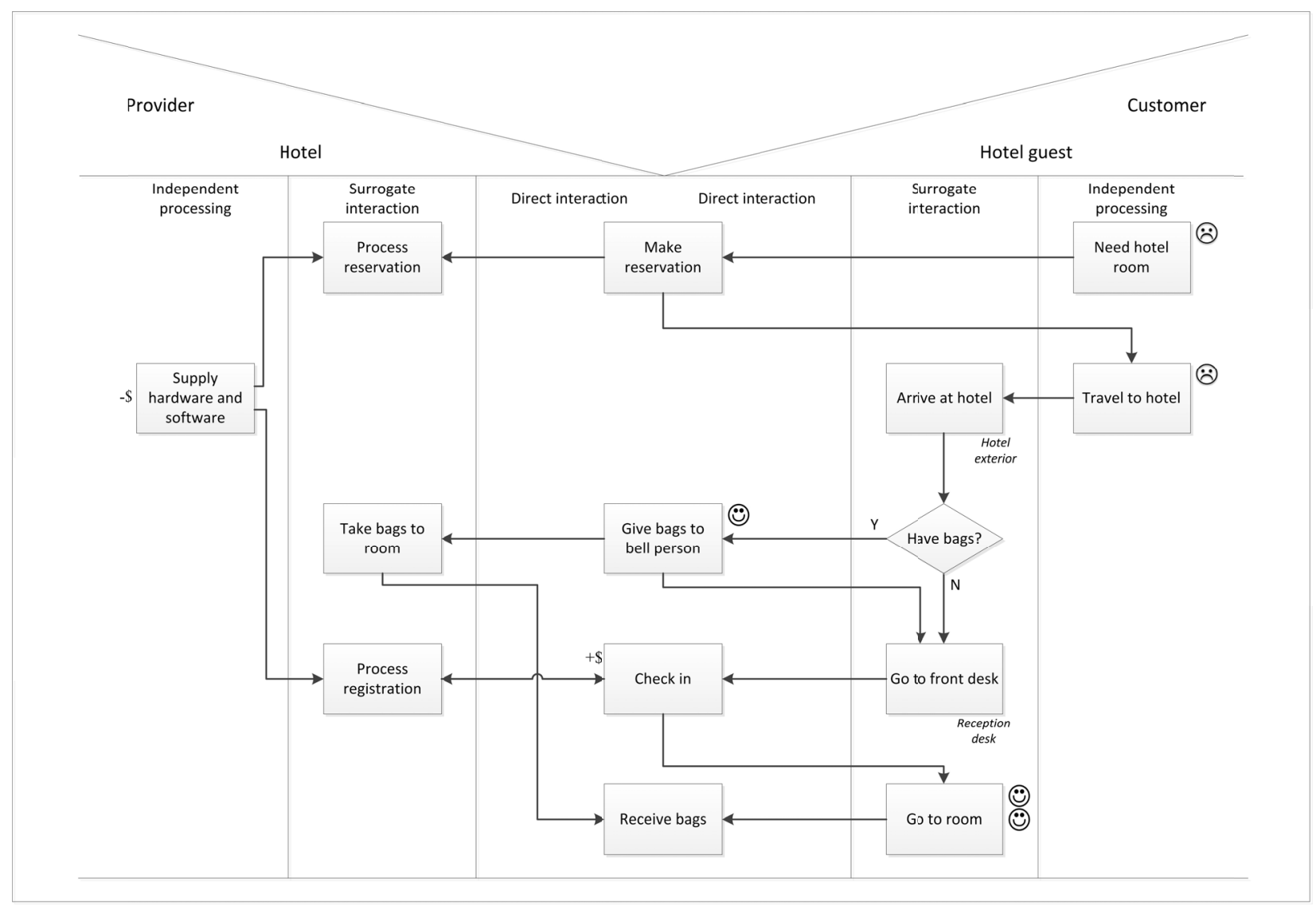

Figure 2. PCN diagram of a hotel stay

Different process entities have diverse needs. Taking into account the kind of need, Sampson (2012b) characterizes two sets of process entity: specific beneficiary and generic beneficiary. A specific beneficiary, in this example 
identified as the customer, is an entity that profits a specific benefit from involvement in a service process. Generic beneficiaries, in this case the hotel, profit generally (for example, money), when participating in a service process.

Each process entity, to a certain degree, has control over the process steps entailed in each process domain within the entity. These domains can be grouped in three divisions/regions: direct interaction region, surrogate interaction region, and Independent processing region. Direct interaction region includes the interactions between entity's employees with another entity's employees. Surrogate interaction region involves an interaction between an entity and any non-human assets of another entity (for example, information or technology). Any process steps of an entity not involving interaction with another entity are included within independent processing region. These regions are in fact based on Wemmerlov's (1990) explanation of three types of contact between customer and provider in a service process. These regions can be utilised by service designers to tailor a service process based on specific preference provided by the client, or to increase process efficiency and economies of scale.

Table 1. Concepts in PCN

\begin{tabular}{|c|c|}
\hline Concept & Definition \\
\hline$<$ Process Step $>$ & A process step represents a step of process chain. \\
\hline$<$ Arrow $>$ & An Arrow represents state dependency and connects process steps to each other. \\
\hline$<$ Process Entity $>$ & $\begin{array}{l}\text { A Process Entity is an entity that participates in and makes decisions about steps of a } \\
\text { process chain. }\end{array}$ \\
\hline$<$ Value $>$ & Value refers to the satisfaction of process entity needs. \\
\hline$<$ Specific Beneficiary $>$ & $\begin{array}{l}\text { A Specific Beneficiary is a process entity that participates in a process chain to have } \\
\text { needs met by the specific competencies in the process chain. }\end{array}$ \\
\hline$<$ Generic Beneficiary $>$ & $\begin{array}{l}\text { A Generic Beneficiary is a process entity that participates in a process chain to acquire } \\
\text { generic resources (money) to meet needs from other process chains. }\end{array}$ \\
\hline$<$ Process Domain $>$ & $\begin{array}{l}\text { A Process Domain is a portion of process chain that falls under a process entity's control } \\
\text { and responsibility. }\end{array}$ \\
\hline $\begin{array}{l}<\text { Direct } \\
\text { Region }>\end{array}$ & $\begin{array}{l}\text { A Direct Interaction Region is the areas of a process domain for process steps involving } \\
\text { person to person interaction between process entities. }\end{array}$ \\
\hline $\begin{array}{l}<\text { Surrogate Interaction } \\
\text { Region }>\end{array}$ & $\begin{array}{l}\text { A Surrogate Interaction Region is the areas of a process domain for process steps } \\
\text { involving interaction with non-human resources of another process entity (e.g. } \\
\text { technology or information). }\end{array}$ \\
\hline $\begin{array}{l}<\text { Independent } \\
\text { Processing Region }>\end{array}$ & $\begin{array}{l}\text { An Independent Processing Region is the areas of a process domain for process steps that } \\
\text { are performed independent from other process entities in the process chain networks. }\end{array}$ \\
\hline $\begin{array}{l}<\text { Nonmonetary Benefits } \\
\text { Tag }>\end{array}$ & $\begin{array}{l}\text { A Nonmonetary Benefits Tag identifies the process steps where customer receives } \\
\text { benefits. }\end{array}$ \\
\hline $\begin{array}{l}<\text { Nonmonetary } \quad \text { Costs } \\
\text { Tag }>\end{array}$ & $\begin{array}{l}\text { A Nonmonetary Costs Tag identifies the process steps where customer incurs } \\
\text { nonmonetary costs. }\end{array}$ \\
\hline $\begin{array}{l}<\text { Monetary } \\
\text { Compensations Tag }>\end{array}$ & $\begin{array}{l}\text { A Monetary Compensations Tag identifies the process steps where the provider receives } \\
\text { monetary compensations. }\end{array}$ \\
\hline$<$ Monetary Costs Tag $>$ & A Monetary Costs Tag identifies the process steps where the provider incurs costs. \\
\hline $\begin{array}{l}<\text { Environmental } \\
\text { Conditions Label }>\end{array}$ & $\begin{array}{l}\text { An Environmental Conditions Label } \\
\text { physical evidence seen by customer. }\end{array}$ \\
\hline
\end{tabular}

Note: Definitions from Sampson (2012a)

The steps of creating a PCN diagram are as follows (Sampson 2012b, p.190 \& 191):

Defining the target service process;

Defining process entities involved in the service process; 
Defining the first and the last process steps;

Defining intermediate process steps and their position in different regions of each process domain; connecting them with arrows to present process steps' dependency.

Tagging the process steps that specific beneficiary receives nonmonetary benefits (tagged (:)) and incurs nonmonetary costs (tagged :);

Tagging the steps that generic beneficiary receives monetary compensations (tagged $+\$$ ) and incurs monetary costs (tagged -\$);

Labelling a number of process steps with a term to illustrate environmental conditions that affect customer service delivery perception. In Figure 2, 'hotel exterior' and 'reception desk' are environmental conditions labels for 'arrive at hotel' and 'go to front desk' process steps.

Table 1 represents the key concepts of $\mathrm{PCN}$ and their precise definitions to complete step two of the research method.

\section{Business Process Modeling Notation (BPMN)}

Within BPMN are both core sets of constructs and extended sets of constructs. Business analysts and non-technical users can use these core sets to model processes and actions for all stakeholders involved (Ko, Lee, \& Lee, 2009; Recker, 2011). Technical users, on the other hand, use the extended set, which allows for more complexity and is useful for thorough design and automation in "workflow engineering, simulation, or web service composition" (Recker 2010, p.183). In this paper, we will consider the core set of BPMN constructs, since we are comparing BPMN with service blueprinting, which is used to communicate with all stakeholders, even those non-technical users. In figure 3 below, a BPMN diagram of the hotel stay example is shown.

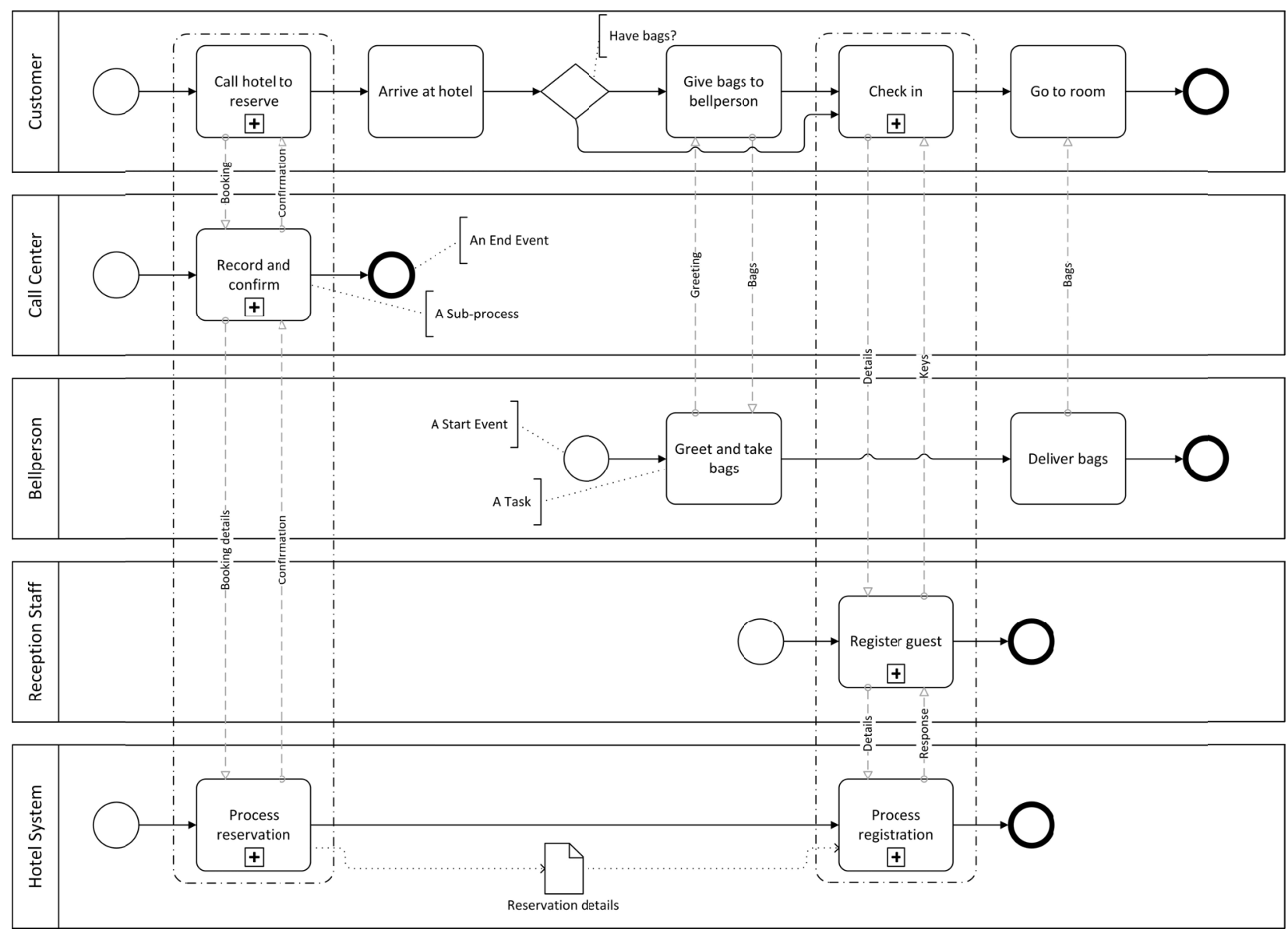

Figure 3. BPMN diagram of a hotel stay 
Table 2. Concepts in BPMN

\begin{tabular}{|c|c|}
\hline Concept & Definition \\
\hline$<$ Activity $>$ & $\begin{array}{l}\text { An Activity is a generic term for work that an organization performs in a process. An activity can } \\
\text { be atomic or compound. An atomic activity (task) is used when the work in the process is not } \\
\text { broken down to a finer level of detail. A compound activity (sub-process) comprising more } \\
\text { compound activities or tasks. }\end{array}$ \\
\hline$<$ Event $>$ & $\begin{array}{l}\text { An Event is something that "happens" during the course of a process. These events affect the flow } \\
\text { of the model and usually have a cause (trigger) or an impact (result). An event can happen at the } \\
\text { start of a process (start event), during a process (intermediate event), or at the end of a process } \\
\text { (end event). }\end{array}$ \\
\hline$<$ Pool $>$ & $\begin{array}{l}\text { A Pool represents a participant in a process. A pool is a graphical container for partitioning a set } \\
\text { of activities from other pools/participants. }\end{array}$ \\
\hline$<$ Lane $>$ & $\begin{array}{l}\text { A Lane is a sub-partition within a pool. Lanes are often used for such things as internal roles (e.g., } \\
\text { manager, associate), systems (e.g., an enterprise application), or an internal department (e.g., } \\
\text { shipping, finance). }\end{array}$ \\
\hline $\begin{array}{l}<\text { Sequence } \\
\text { Flow }>\end{array}$ & $\begin{array}{l}\text { A Sequence Flow is used to show the order that activities will be performed in a process by a } \\
\text { participant. Sequence flows are connecting and ordering activities within a pool. }\end{array}$ \\
\hline $\begin{array}{l}<\text { Message } \\
\text { Flow }>\end{array}$ & $\begin{array}{l}\text { A Message Flow is used to show the flow of messages between two participants that are prepared } \\
\text { to send and receive them. In BPMN, two separate pools in a diagram represent the two } \\
\text { participants. }\end{array}$ \\
\hline$<$ Message $>$ & A Message is used to depict the contents of a communication between two participants. \\
\hline$<$ Gateway $>$ & $\begin{array}{l}\text { A Gateway is used to control the divergence and convergence of sequence flows in a process. } \\
\text { Thus, it will determine traditional decisions, as well as the forking, merging, and joining of paths. }\end{array}$ \\
\hline$<$ Data Object $>$ & $\begin{array}{l}\text { Data Objects provide information about what activities require to be performed and/or what they } \\
\text { produce. }\end{array}$ \\
\hline $\begin{array}{l}<\text { Text } \\
\text { Annotation }>\end{array}$ & $\begin{array}{l}\text { Text Annotations are a mechanism for a modeller to provide additional information for the reader } \\
\text { of a BPMN diagram. }\end{array}$ \\
\hline$<$ Association $>$ & $\begin{array}{l}\text { An Association is used to link artefacts (data objects and annotations) to flow objects (activities, } \\
\text { events and gateways). }\end{array}$ \\
\hline$<$ Group $>$ & $\begin{array}{l}\text { A Group identifies logically related activities and does not affect the sequence flow. The grouping } \\
\text { can be used for documentation or analysis purposes. }\end{array}$ \\
\hline
\end{tabular}

Note: Definitions from OMG (2011)

Within BPMN, there are four groups of basic constructs. These are, namely, flow objects, connecting objects, swimlanes and artefacts. Flow objects include event, activity and gateway, and connecting objects include sequence flow, message flow and association. Swimlanes include pools and lanes. Artefacts contain data object, group and text annotation.

A circle in a BPMN diagram indicates an event triggering activity. Three types of trigger exist: start event (when a participant begins a process), intermediate event (happens at the middle of a process) and end event (when a participant finishes a process). An event that happens during a process at anytime affecting the flow is known as a super-type event.

Any actions in processes are denoted by activity. These can be atomic (a specific task that won't be broken down) or compound (contain a sub-process). Rounded rectangles in BPMN diagrams represent activities. If these activities are complex, they are shown with a plus sign in the centre and the bottom of the activity rectangle.

Divergence and convergence of different process flows is possible through a gateway. There are six basic types of splitting and joining process flows defined by Object Management Group (2011); these being represented as diamonds differentiated by the markings inside them. We define a super-type gateway as one allowing for the branching, forking, merging or joining of paths: "whether exactly one (exclusive "or"), more than one ("or"), or all 
("and") of the activities entering or leaving the join or split, respectively, are required prior to (join) or must follow (split)" (Milton and Johnson 2012, p.612). In the hotel stay example, depending on whether the customer has bags or not, he will either give these to the bellperson or go for check-in respectively.

The sequence of activities performed in a sequence flow is indicated by a solid arrow. A Message flow shows a message running across different activities and is denoted by dashed lines with an arrow indicating the direction of communication. The label on the arrow shows the type of communication.

Two formal grouping concepts are defined within BPMN: pool and lane. A participant group is represented by pool. These can either be a partner (e.g., a company), a specific partner role (e.g., a buyer, seller, or manufacturer) or a group internal to the company (OMG, 2011). Activities within a pool are categorized using lanes. These can either represent an internal role, an internal department, or a system. Process designers can utilise these pool and lane constructs in different ways; much freedom being allowed for in their respective use. It is essential that one knows that sequence flows connect activities within a pool and message flows connect activities between different pools.

Dotted lines with arrows in BPMN diagrams represent associations, and these connect artefacts with flow objects. The direction of the arrow shows the direction of the association. Any data that is required for, or produced by, an activity with an association linking to activities is shown by a data object.

Any activities that are logically connected for analysis or documentation purposes are linked by groups. These groups are denoted by a dashed-dotted box around the activities. In our example regarding the hotel stay, there are two sets of grouped activities. These are, namely, reservation sub-processes and registration sub-processes.

Any comments that are to be included for readers are shown by text annotations. In the hotel stay example, different representational symbols for tasks, sub-processes, start events and end events are shown by annotations. The core concepts of BPMN along with their definitions are shown in Table 2. The next section presents the conceptual evaluation of BPMN against service blueprinting.

\section{Conceptual Evaluation of BPMN against PCN}

Table 3. Results of conceptual evaluation of BPMN against PCN

\begin{tabular}{ccc}
\hline Concepts of PCN & Degree of overlap & Supportive concepts of BPMN \\
\hline$<$ Process Step $>$ & $\checkmark$ & $<$ Activity $>$ \\
$<$ Arrow $>$ & $\checkmark$ & $<$ Sequence Flow plus Message Flow $>$ \\
$<$ Process Entity $>$ & $\checkmark$ & $<$ Pool $>$ \\
$<$ Value $>$ & $\mathbf{x}$ & \\
$<$ Specific Beneficiary $>$ & $\mathbf{x}$ & \\
$<$ Generic Beneficiary $>$ & $\mathbf{x}$ & $<$ Pool $>$ \\
$<$ Process Domain $>$ & $\checkmark$ & \\
$<$ Direct Interaction Region $>$ & $\mathbf{x}$ & \\
$<$ Surrogate Interaction Region $>$ & $\mathbf{x}$ & \\
$<$ Independent Processing Region $>$ & $\mathbf{x}$ & \\
$<$ Nonmonetary Benefits Tag $>$ & $\mathbf{x}$ & \\
$<$ Nonmonetary Costs Tag $>$ & $\mathbf{x}$ & \\
$<$ Monetary Compensations Tag $>$ & $\mathbf{x}$ & \\
$<$ Monetary Costs Tag $>$ & $\mathbf{x}$ & \\
$<$ Environmental Conditions Label $>$ & $\mathbf{x}$ & \\
\hline
\end{tabular}

Table 3 shows the results of the conceptual evaluation of BPMN, using PCN concepts as a benchmark. The results indicate that $<$ Process Step $>,<$ Arrow $\rangle$, $<$ Process Entity $>$ and $<$ Process Domain $>$ are covered completely by different supportive BPMN concepts. Other concepts of PCN, including $\langle$ Value $\rangle,\langle$ Specific Beneficiary $\rangle,\langle$ Generic Beneficiary $>,<$ Direct Interaction Region $>,<$ Surrogate Interaction Region $>,<$ Independent Processing Region $>$, 
$<$ Customer Benefits Tag $>,<$ Nonmonetary Costs Tag $>,<$ Monetary Compensations Costs Tag $>,<$ Monetary Costs Tag $>$ and $<$ Environmental Conditions Label> are not covered at all by any BPMN concepts. The following paragraphs explain the results utilizing semiotic theory to discuss how semantic fields of PCN concepts are covered (or not covered) by supportive BPMN concepts according to the represented order of concepts in table 3 .

Sampson (2012a, p.19; 2012b, p.186) defines a service process in PCN as "a sequence of steps". Indeed $<$ Process Step $>$ is the most basic concept in a PCN diagram. In BPMN, <Activity $>$ does the same job; it is one of the basic elements in BPMN diagrams along with $<$ Event $>$ and $<$ Gateway $>$ (Recker, 2011; White, 2004).

A $<$ Process Step $>$ in a PCN diagram sometimes consists of two or more $<$ Process Step $>$ concepts from different participant entities. This happens when a designer depicts a $<$ Process Step $>$ which involves the interaction of provider employees with a customer. This kind of $<$ Process Step $>$ always falls in the $<$ Direct Interaction Region $>$. Therefore, a BPMN diagram does not show this kind of $<$ Process Step $>$ as it is not possible that an activity in a BPMN diagram passes $<$ Pool $>$ boundaries. This problem can be solved by considering this kind of $<$ Process Step $>$ as two separate $<$ Process Step $>$ concepts, one falling under $<$ Direct Interaction Region $>$ of provider and the other one falling under $<$ Direct Interaction Region $>$ of customer.

An $<$ Arrow $>$ connects process steps to show that a process step is dependent on the state being of resources of another process step (Sampson, 2012a, 2012b). BPMN includes two different connecting flows to represent connections between activities: <Sequence Flow $>$ and $<$ Message Flow $>$. A $<$ Sequence Flow $>$ orders activities performed by a participant. A $<$ Message Flow $>$ represents the flow of communication between activities performed by two different participants. However, an $<$ Arrow $>$ connects the activities in a PCN diagram regardless of whether they are performed by a specific participant or two different participants. Consequently, $<$ Sequence Flow plus Message Flow $>$ from BPMN covers $<$ Arrow $>$ in PCN.

A $<$ Process Entity $>$ is a participant entity in a process that performs and makes decisions about a set of process steps in a process. There are two types of $<$ Process Entity $>$ concepts in this study: customer $<$ Process Entity $>$ and provider $<$ Process Entity $>$. $<$ Pool $>$ is used to show different participants that are involved in a service delivery process. Object Management Group (2011, p.112) states "a Participant can be a specific PartnerEntity (e.g., a company) or can be a more general PartnerRole (e.g., a buyer, seller, or manufacturer)". BPMN can employ $<$ Pool $>$ concepts to represent the provider and the customer. Consequently, $<$ Process Entity $>$ in PCN can be supported by $<$ Pool $>$ from service blueprinting.

The concepts of $<$ Value $>,<$ Specific Beneficiary $>$ and $<$ Generic Beneficiary $>$ are not supported by BPMN. Recall from section 3; <Value $>$ is defined as "the satisfaction of process entity needs" (Sampson 2012a, p.28). In fact, gaining $<$ Value $>$ is the motivation of each entity to participate in a service delivery process. If an entity's need can be met by receiving some kind of benefit other than money (e.g. a customer who receives consultancy advices) that entity is a $<$ Specific Beneficiary $>$ (Sampson, 2012a, 2012b). We know the customer is a $<$ Specific Beneficiary $>$ as he/she participates in a service process to gain a specific competency other than money. <Generic Beneficiary $>$ involves in a process to receive generic benefits such as money which can be used to satisfy its needs as a customer or consumer in other process chains (Sampson, 2012a, 2012b). We know a provider is usually the $<$ Generic Beneficiary $>$. BPMN does not support $<$ Value $>$, $<$ Specific Beneficiary $>$ and $<$ Generic Beneficiary $>$ of PCN since there is no mechanism to identify what the needs are for each participant entity in the service process.

$<$ Process Domain $>$ is the final PCN concept in table 3, which is completely covered by BPMN concepts. According to Sampson (2012c, p.187) < Process Domain > is "the set of process steps that are initiated, led, performed, and, to some degree, controlled by process entity." Recall from PCN explanation section (section 3.3), $<$ Process Domain $>$ is defined as "a portion of process chain that falls under an entity's control and responsibility" (Sampson, 2012a). A $<$ Pool $>$ in a BPMN diagram categorizes activities that a participant entity has control over and works as an operant resource on them. Therefore, $<$ Pool $>$ in BPMN completely supports $<$ Process Domain $>$ in PCN.

PCN defines three process regions: $<$ Direct Interaction Region $>,<$ Surrogate Interaction Region $>$ and $<$ Independent Processing Region $>$. $<$ Direct Interaction Region $>$ includes the process steps involving provider employee interactions with customers. $<$ Surrogate Interaction Region $>$ defined as "Areas of a process domain for steps involving interaction with non-human resources of another entity (e.g. technology or information)" (Sampson 2012a, p.28). $<$ Independent Processing Region $>$ includes process steps which involve only one $<$ Process Entity $>$ and there is no interaction with another $<$ Process Entity $>$ (Sampson, 2011, 2012a, 2012b). BPMN does not provide any concepts to differentiate activities based on the nature of interaction. Consequently, BPMN does not support process regions in service blueprinting. 
There are four concepts in PCN to identify value propositions of each process step: $<$ Nonmonetary Benefits Tag $>$, $<$ Nonmonetary Costs Tag $>$, $<$ Monetary Costs Tag $>$ and $<$ Monetary Compensations Tag $>$. Explained in section 3.3, $<$ Nonmonetary Benefits Tag $>$ is used to identify process steps where a $<$ Specific Beneficiary $>$ receives nonmonetary benefits (e.g. when pizza is delivered to a customer). $<$ Nonmonetary Costs Tag $>$ is used when a $<$ Specific Beneficiary $>$ incurs nonmonetary costs (e.g. when a customer travels to a pizza restaurant). $<$ Monetary Compensations Tag $>$ is used to identify process steps that $<$ Generic Beneficiary $>$ receives money (e.g. when a pizza restaurant employee collects payment). $<$ Monetary Costs $\mathrm{Tag}>$ is used to identify process steps that $<$ Generic Beneficiary $>$ incurs monetary costs (e.g. when a restaurant prepares ingredients) (Sampson, 2012a). PCN designers employ these concepts to understand where value potentials are created and where value realizations occur. The transformation of value happens through the preparation of resources which have value potential during a process step and realization of value happens by performing some other steps (Grönroos, 2008). BPMN does not identify if there are nonmonetary or monetary benefits and costs for each activity.

The last concept in Table 3 is $<$ Environmental Conditions Label $>$. This is an optional concept to identify physical evidences for each process step which affects customer service perception (Sampson, 2011, 2012a, 2012b). BPMN does not support $<$ Environmental Conditions Label $>$ in PCN.

The next section presents indicative results of conceptual evaluation of PCN against BPMN in a tabular form. Then, the natures of the gaps in coverage are discussed in detail.

\section{Conceptual Evaluation of PCN against BPMN}

Table 4. Results of conceptual evaluation of PCN against BPMN

\begin{tabular}{ccc}
\hline Concepts of BPMN & Degree of overlap & Supportive concepts of PCN \\
\hline$<$ Activity $>$ & $\checkmark$ & $<$ Process Step $>$ \\
$<$ Event $>$ & $\mathbf{x}$ & \\
$<$ Pool $>$ & $\checkmark \mathrm{p}$ & $<$ Process Entity plus Process Domain $>$ \\
$<$ Lane $>$ & $\mathbf{x}$ & \\
$<$ Sequence Flow $>$ & $\checkmark$ & $<$ Arrow $>$ \\
$<$ Message Flow $>$ & $\checkmark$ & $<$ Arrow $>$ \\
$<$ Message $>$ & $\mathbf{x}$ & \\
$<$ Gateway $>$ & $\mathbf{x}$ & \\
$<$ Data Object $>$ & $\mathbf{x}$ & \\
$<$ Text Annotation $>$ & $\mathbf{x}$ & \\
$<$ Association Flow $>$ & $\mathbf{x}$ & \\
$<$ Group $>$ & $\mathbf{x}$ & \\
\hline
\end{tabular}

Table 4 shows the results of the conceptual evaluation of PCN, using BPMN concepts as a benchmark. The results show complete coverage of $\langle$ Activity $\rangle$, $<$ Sequence Flow $>$ and $<$ Message Flow $>$ from BPMN by different concepts from PCN. In addition, the table indicates that $<$ Pool $>$ is covered partially by PCN. Finally, there is no coverage for $\langle$ Event $>,\langle$ Lane $>,<$ Message $>,<$ Gateway $>,\langle$ Data Object $>,<$ Text Annotation $>,<$ Association Flow $\rangle$ and $<$ Group $>$. The following paragraphs utilize semiotic theories to discuss the results in detail according to the table order.

An <Activity $>$ is defined by Object Management Group (2011, p.29) as "work that a company performs in a process". An $<$ Activity $>$ can be atomic (task) or can be divided into more than one $<$ Activity $>$ (compound). In a similar way, A $<$ Process Step $>$ is work that is done by a participant entity in a PCN diagram; sometimes a $<$ Process Step $>$ can be divided into more than one $<$ Process Step $>$ when it is needed. Consequently, the $<$ Process Step $>$ concept from PCN covers the $<$ Activity $>$ concept from BPMN.

An $<$ Event $>$ is defined as "something that happens during the course of a Process. These Events affect the flow of the model and usually have a cause (trigger) or an impact (result). There are three types of Events, based on when they affect the flow: Start, Intermediate, and End" (OMG 2011, p.29). PCN does not include any similar concepts to cover the $<$ Event $>$ concept in BPMN.

A $<$ Pool $>$ is used to categorize activities based on different participants involved in a process. Particularly, a $<$ Pool $>$ can be used to show customer activities and provider activities. A $<$ Pool $>$ can categorize a provider's internal department, system, or role as well. PCN identifies provider and customers as a $<$ Process Entity $>$ and considers the 
activities performed and controlled by them as their $<$ Process Domain $>$. Therefore, $<$ Process Entity plus Process Domain $>$ from PCN partly covers $<$ Pool $>$ from BPMN. A $<$ Lane $>$ is used to categorize activities in a process to represent an internal role, system, or department; it is a sub-partition within a $<$ Pool $>$. PCN does not define any concepts to show internal roles, systems, or internal departments of a provider.

In BPMN diagrams, a $<$ Sequence Flow $>$ orders activities in a $<$ Pool $>$ and a $<$ Message Flow $>$ connects activities from one $<$ Pool $>$ to another one. A $<$ Message $>$ is used to show the content of $<$ Message Flow $>$ between two activities. An $<$ Arrow $>$ in PCN presents state dependency and indicates that a process step is dependent on some resource being in a state provided by another process step. Sampson (2012a, 2012c) states that an $<$ Arrow $>$ can show the movement of goods and information between activities as well. An $<$ Arrow $>$ can connect customer activities, provider activities, or a customer activity to a provider activity. As a result, we conclude that $<$ Arrow $>$ from PCN covers $<$ Sequence Flow $>$ and $<$ Message Flow $>$ from BPMN. PCN does not provide any concept to show the content of connections between different participants.

A $<$ Gateway $>$ is used to branch, fork, merge, or join the paths in a BPMN diagram. The investigations show that whilst Sampson (2012a, 2012b, 2011) used in a number of PCN diagrams a diamond shaped symbol for branching paths based on a decision, he did not define any concepts for this purpose. Therefore, BPMN $<$ Gateway $>$ is not supported by any concepts from PCN.

BPMN includes three artefacts: $<$ Data Object $>$, $<$ Text Annotation $>$ and $<$ Group $>$. A $<$ Data Object $>$ represents required or produced data by an activity. A $<$ Data object $>$ does not affect the sequence of activities by participants or flow of messages between participants. A < Text Annotation> is defined by Object Management Group (2011, p.30) as "a mechanism for a modeller to provide additional text information for the reader of a BPMN Diagram". Specifically, <Text Annotation $>$ is used to provide additional information about flow objects. Recker et al. (2010; 2011) reports that $<$ Text Annotation $>$ is used also to represent business rules. BPMN employs $<$ Association Flow $>$ to associate a $<$ Data Object $>$ to an activity. An $<$ Association Flow $>$ is used also to associate a $<$ Text Annotation $>$ to a flow object. PCN does not define any concepts regarding data input and output of process steps. In addition, PCN does not define any concepts to add additional information about different represented concepts in a PCN diagrams. Therefore, PCN does not cover the BPMN concepts of $<$ Data Object $>$, $<$ Text Annotation $>$ and $<$ Association Flow $>$. $<$ Group $>$ categorizes $<$ Activity $>$ concepts; it does not affect the sequence of process elements. $<$ Group $>$ is used for the purpose of documentation and process analysis. Sampson (2012a) did not define any concepts for the identification of a particular type of process step. Therefore, there are no corresponding concepts for $<$ Group $>$ between PCN concepts.

The following sections discuss the findings from both comparisons and sconclusions.

\section{Findings}

The results of conceptual evaluations indicate that $<$ Process Step $>$ from PCN covers the same semantic field as $<$ Activity $>$ in BPMN. $<$ Arrow $>$ from PCN connects $<$ Process Step $>$ concepts based on their state dependency rather than the performing actor, so it is fully covered by $<$ Sequence Flow plus Message Flow $>$ from BPMN. On the other hand, $<$ Arrow $>$ fully supports $<$ Sequence Flow $>$ and $<$ Message Flow $>$. Therefore, $<$ Arrow $>$ covers the same semantic field as $<$ Sequence Flow plus Message Flow $>$.

$<$ Pool $>$ and $<$ Lane $>$ from BPMN have a capacity to categorize activities in a process in many different ways based on participant entities, internal roles, systems, and internal departments of an organization. A $<$ Process Entity $>$ is a participant entity in a process and it has a $<$ Process Domain $>$ which consists of the activities under the control of that participant entity. A $<$ Pool $>$ can identify a participant entity in a service process and partition its activities from other participant entities. Consequently, $<$ Pool $>$ from BPMN has a broader semantic field than $<$ Process Entity plus Process Domain $>$. In contrast, PCN categorizes a participant entity's process steps based on the nature of its interaction with other participant entities in a service process to three groups: <Direct Interaction Region>, $<$ Surrogate Interaction Region $>$ and $<$ Independent Processing Region $>$. $<$ Direct Interaction Region $>$ consists of activities that involve the interaction between human resources of two participant entities. $<$ Surrogate Interaction Region $>$ consists of activities involving a participant entity interacting with nonhuman resources (e.g. information, technologies or goods) of another participant entity. <Independent processing Region> consists of activities that are independently performed by a single participant entity. BPMN cannot do this at all as it categorizes activities based on their performer.

Another major difference between these two process modeling methods is found when the concept of $\langle$ Value $\rangle$ in PCN is compared with BPMN concepts. PCN defines $\langle$ Value $\rangle$ to explain why participant entities are engaged to involve in a service delivery process. To understand the reason and motivation of participant entities' involvement in a service process, value propositions must be identified. To do this, PCN defines six concepts: $<$ Specific Beneficiary $>,<$ Generic Beneficiary $>$, $<$ Nonmonetary Benefits Tag $>,<$ Nonmonetary Costs tag $>,<$ Monetary Compensations tag $>$ and $<$ Monetary Costs Tag $>$. $<$ Specific Beneficiary $>$ and $<$ Generic Beneficiary $>$ are defined to 
understand the ultimate goals of participant entities in a service process: gaining specialized competencies or generic benefits (e.g. money). After identifying $<$ Specific Beneficiary $>$ and $<$ Generic Beneficiary $>$, PCN analysts employ the concepts of $<$ Nonmonetary Benefits Tag $>$, $<$ Nonmonetary Costs Tag $>,<$ Monetary Compensations Tag $>$ and $<$ Monetary Costs Tag $>$ to identify the $<$ Process Step $>$ concepts that contribute to the realization of $<$ Value $\rangle$. In comparison, BPMN does not consider why participant entities are involved in a service process and BPMN does not define any concepts regarding the goals and motivations of participant entities.

PCN does not cover $<$ Data Object $>$, $<$ Text Annotation $>$ and $<$ Group $>$ from BPMN. These BPMN artefacts are defined to add additional information about flow objects. BPMN artefacts do not affect the basic structure of the service process diagram. Having artefacts enables BPMN to show more details about how the process is performed and suggests BPMN provides more concepts compared with PCN for the purpose of service process documentation.

\section{Conclusions}

The results and findings imply that PCN has fundamentally a different view with BPMN towards the categorization of activities. PCN differentiates between process steps based on their nature of interaction to understand how the provider can reconfigure process steps across each process region and improve the performance of service processes. PCN introduces the concept of $\langle$ Value $\rangle$ and a series of related concepts to help service process designers and analysts to understand what the benefits and costs of each process step are in detail. These indicate that PCN is a better process modeling method for service process analysis and improvement in comparison with BPMN considering managerial principles of process efficiency, economies of scale, and customization.

In comparison, BPMN categorizes activities in a way to represent organizational responsibilities and communications between participant entities (especially customer and provider), organizational departments, systems, and roles. Defining a series of artefacts in BPMN also implies an informational view of BPMN and suggests that BPMN has a higher capability than PCN to add additional information about concepts when it is needed.

\section{Implications for Practice}

Even though PCN does not demonstrate organizational roles and responsibilities, it provides a useful representation of all customer process steps and process steps across the organization. Using PCN, service designers can implement service operations management ideologies (process efficiency, economies of scale and customization) and reposition process steps in order to create process innovation, whilst taking account for the costs and benefits of each step.

Contrastingly, BPMN can be used when the practitioners aim to depict organizational departments, systems, and roles that are involved in service delivery processes with a larger complexity. Also, BPMN has the ability to portray any parts of the service provider organization and customer interaction. A BPMN diagram is also useful in representing inter-organizational communications. Such organizational and informational views make BPMN the preferred modeling approach for information systems analysts and professionals.

\section{Future Research}

It is possible for future studies to assess each process modeling technique pragmatically, in order to examine two sides of each modeling equation: practice and interpretation. To conduct studies on modeling practice, one would ask a number of users of these models to understand in what way they used the concepts in each of these models to visualize numerous phenomena. Subsequently, the researcher would employ a number of case studies to examine, for example, which stage of service development process and for what purposes these models were used, and how practitioners used these models in conjunction (modeling interpretation).

\section{References}

BPMI.org. (2004). Business Process Modeling Notation (BPMN).

Cruse, D. A. (2004). Meaning in language: an introduction to semantics and pragmatics. Oxford: Oxford University Press.

Culler, J. (1976). Saussure. Glasgow, UK: Fontana/Collins.

Decker, G., Kopp, O., Leymann, F., \& Weske, M. (2009). Interacting services: from specification to execution. Data \& Knowledge Engineering, 68(10), 946-972. http://dx.doi.org/10.1016/j.datak.2009.04.003

Dijkman, R. M., Dumas, M., \& Ouyang, C. (2008). Semantics and analysis of business process models in BPMN. Information and Software Technology, 50(12), 1281-1294. http://dx.doi.org/10.1016/j.infsof.2008.02.006

Eco, U. (1976). A theory of semiotics. Midland, Bloomington.

Grönroos, C. (2008). Service logic revisited: who creates value? And who co-creates? European Business Review, 20(4), 298-314. http://dx.doi.org/10.1108/09555340810886585 
Kazemzadeh, Y., Milton, S. K., \& Johnson, L. W. (in press-a). A comparison of concepts in service blueprinting and process chain network (PCN). International Journal of Business and Management.

Kazemzadeh, Y., Milton, S. K., \& Johnson, L. W. (in press-b). Service blueprinting and business process modeling notation (BPMN): a conceptual comparison. Asian Social Science.

Kazemzadeh, Y., Milton, S. K., \& Johnson, L. W. (in press-c). Service blueprinting and process-chain-network (PCN): an ontological comparison. International Journal of Qualitative Research in Services.

Ko, R. K. L., Lee, S. S. G., \& Lee, E. W. (2009). Business process management (BPM) standards: a survey. Business Process Management Journal, 15(5), 744-791. http://dx.doi.org/10.1108/14637150910987937

Milton, S. K., \& Johnson, L. W. (2012). Service blueprinting and BPMN: a comparison. Managing Service Quality, 22(6), 606-621. http://dx.doi.org/10.1108/09604521211287570

Milton, S. K., \& Kazmierczak, E. (2004). An ontology of data modelling languages. Journal of Database Management, 15(2), 19-38.

OMG. (2011). Business Process Model and Notation (BPMN).

Rabhi, F. a., Yu, H., Dabous, F. T., \& Wu, S. Y. (2006). A service-oriented architecture for financial business processes. Information Systems and E-Business Management, 5(2), 185-200. http://dx.doi.org/10.1007/s10257-006-0041-x

Recker, J. (2010). Opportunities and constraints: the current struggle with BPMN. Business Process Management Journal, 16(1), 181-201. http://dx.doi.org/10.1108/14637151011018001

Recker, J. (2011). Evaluations of process modeling grammars. In Lecture Notes in Business Information Processing. Springer-Verlag Berlin Heidelberg.

Recker, J., Indulska, M., Rosemann, M., \& Green, P. (2010). The ontological deficiencies of process modeling in practice. European Journal of Information Systems, 19(5), 501-525. http://dx.doi.org/10.1057/ejis.2010.38

Recker, J., Rosemann, M., Green, P., \& Indulska, M. (2011). Do ontological deficiencies in modeling grammars matter? MIS Quarterly, 35(1), 57-79.

Sampson, S. E. (2011). Introduction to PCN analysis 1 (Vol. 15, pp. 1-16). Provo, Utah, USA.

Sampson, S. E. (2012a). Essentials of service design (Second Edi.). Provo, Utah, USA: Brigham Young University.

Sampson, S. E. (2012b). Visualizing service operations. Journal of Service Research, 15(2), 182-198. http://dx.doi.org/10.1177/1094670511435541

Wemmerlöv, U. (1990). A taxonomy for service processes and its implications for system design. International Journal of Service Industry Management, 1(3), 20-40.

White, S. A. (2004). Introduction to BPMN. IBM Cooperation, 2(0), 1-11. 